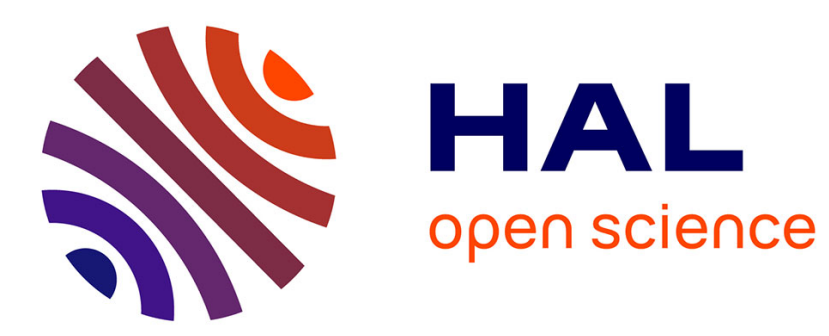

\title{
Relaxing the roles of corners in BDDC by perturbed formulation
}

\author{
Santiago Badia, Hieu Nguyen
}

\section{To cite this version:}

Santiago Badia, Hieu Nguyen. Relaxing the roles of corners in BDDC by perturbed formulation. 2016 . hal-01337852

\section{HAL Id: hal-01337852 \\ https://hal.science/hal-01337852}

Preprint submitted on 27 Jun 2016

HAL is a multi-disciplinary open access archive for the deposit and dissemination of scientific research documents, whether they are published or not. The documents may come from teaching and research institutions in France or abroad, or from public or private research centers.
L'archive ouverte pluridisciplinaire HAL, est destinée au dépôt et à la diffusion de documents scientifiques de niveau recherche, publiés ou non, émanant des établissements d'enseignement et de recherche français ou étrangers, des laboratoires publics ou privés. 


\title{
Relaxing the roles of corners in BDDC by perturbed formulation
}

\author{
Santiago Badia ${ }^{1,2}$ and Hieu Nguyen ${ }^{2}$
}

\section{Introduction}

The Balancing Domain Decomposition by Constraints (BDDC) method was first introduced by Dohrmann [2003]. Compared to its parent, the BDD method by Mandel [1993], one of the advances in BDDC method is the use of constraints to enforce equality of averages across faces, edges, or at individual dofs on substructure boundaries called corners. These constraints serve two purposes. First, they ensure that the coefficient matrix of the coarse problem is always invertible. Second, they induce a natural coarse space leading to fast convergence. While corner constraints do not have significant contribution in serving the second purpose, they are mainly responsible for the first one. In addition, in order to use positive definite sparse direct solvers, which are faster and more robust than their indefinite counterparts, the corners should be chosen so that the local matrix sub-assembled for all dofs in each substructure except corners is positive definite. Here we do not consider a change of basis, cf. Li and Widlund [2006], as it destroys good sparsity pattern of local matrices and is more complicated to implement.

Different corner selection algorithms have been proposed by Dohrmann [2003], Lesoinne [2003], Klawonn and Widlund [2006], Śístek et al. [2012] to guarantee such choices of corners. However, based on our experience, the implementation of this type of algorithms is an involved and time-consuming task, which does depend on the physical problem to be solved and also the type of FE formulation being used. Furthermore, the situation becomes far more complicated when subdomains are disconnected, or only connected by corners or edges. Unfortunately, the currently available parallel mesh parti-

1 Universitat Politecnica de Catalunya, Jordi Girona 1-3, Edifici C1, 08034 Barcelona, Spain.

2 CIMNE - Centre Internacional de Metodes Numerics en Enginyeria, Parc Mediterrani de la Tecnologia, UPC, Esteve Terradas 5, 08860 Castelldefels, Spain. \{sbadia, hnguyen\}@cimne.upc. edu 
tioners, ParMETIS by Karypis et al. [1997] and PT-Scotch by Chevalier and Pellegrini [2008], cannot guarantee connected subdomains.

In this paper, we present a perturbed formulation of the BDDC method where the coarse coefficient matrix and the local stiffness matrices are guaranteed to be positive definite. For this new formulation, corner constraints are optional and should be selected only for convergence purpose. Consequently, one can consider much smaller coarse problems, only involving faces and/or edges. This is particularly important when dealing with unstructured meshes and partitions generated by mesh partitioners, due to the proliferation of corners. Since the coarse problem is the bottleneck that can destroy scalability, these strategies are better suited for large scale simulations.

The presentation of this paper is concise, engineering-friendly and useful to quickly absorb the of essential ideas of the method for implementation. For a full mathematical treatment with complete analysis and additional numerical experiments, we refer the reader to Badia and Nguyen [submitted].

\section{BDDC Overview}

Even though our results do apply for linear elasticity, our presentation, due to limited space, only features Poisson's equation: find $u(x) \in H_{0}^{1}(\Omega)$, for a given polygonal (polyhedral) domain $\Omega \subset \mathbb{R}^{n}, n=2,3$ and a source term $f(x) \in L^{2}(\Omega)$, such that

$$
\underbrace{\int_{\Omega} \nabla u(x) \cdot \nabla v(x) d x}_{\equiv a(u, v)}=\underbrace{\int_{\Omega} f(x) v(x) d x}_{\equiv(f, v)}, \quad \text { for all } v(x) \in H_{0}^{1}(\Omega) .
$$

Let $\mathcal{T}_{h}$ be a shape-regular mesh of size $h$ of $\Omega$. Discretizing (1) using the space $V_{h} \subset H_{0}^{1}(\Omega)$ of linear piecewise polynomials defined on $\mathcal{T}_{h}$, we arrive at the following system of equations:

$$
A u=f .
$$

Let us also consider a nonoverlapping partition of $\Omega$ into subdomains, also known as substructures, $\bar{\Omega}=\cup_{j=1}^{J} \bar{\Omega}_{j}$ with the inter-subdomain interface $\Gamma=\cup_{j=1}^{J} \partial \Omega_{j} \backslash \partial \Omega$. We assume that the partition is quasi-uniform, and the subdomains are obtained by aggregation of elements in $\mathcal{T}_{h}$. We denote $H_{i}$, or generically $H$, the size of $\Omega_{i}$.

Let $K^{(i)}$ be the stiffness matrix associated with substructure $\Omega_{i}$. It should be noted that $K^{(i)}$ is symmetric positive semidefinite and is singular when $\Omega_{i}$ is a floating subdomain $\left(\partial \Omega_{i} \cap \partial \Omega=\emptyset\right)$.

Denote by $R_{i}$ the global to local mapping that restrict any vector $u$ to its local counterpart $u_{i}$, i.e., $u_{i}=R u$. It follows that 


$$
A=R^{T} K R, \quad \text { where } R=\left[R_{1}^{T} \ldots R_{N}^{T}\right]^{T}, K=\operatorname{diag}\left(K^{(1)}, \ldots, K^{(N)}\right) .
$$

For simplicity, we assume that interior dofs are always ordered before interface dofs, namely

$$
u=\left[\begin{array}{ll}
u_{I}^{T} & u_{\Gamma}^{T}
\end{array}\right]^{T}, \quad u_{I}=R_{I} u, \quad u_{\Gamma}=R_{\Gamma} u .
$$

This leads to the following reordered block structures

$$
A=\left[\begin{array}{ll}
A_{I I} & A_{I \Gamma} \\
A_{\Gamma I} & A_{\Gamma \Gamma}
\end{array}\right], \quad K=\left[\begin{array}{cc}
A_{I I} & K_{I \Gamma} \\
K_{\Gamma I} & K_{\Gamma \Gamma}
\end{array}\right], \quad \text { and } \quad K^{(i)}=\left[\begin{array}{cc}
A_{I I}^{(i)} & A_{I \Gamma}^{(i)} \\
A_{\Gamma I}^{(i)} & K_{\Gamma \Gamma}^{(i)}
\end{array}\right] .
$$

The BDDC preconditioner for solving the linear system (2) is completely defined by a weight matrix $W=\operatorname{diag}\left(W^{(1)}, \ldots, W^{(N)}\right)$ and a constraint matrix $C$. The matrix $W$ forms a partition of unity, namely

$$
R^{T} W R=\sum_{i=1}^{N} R_{i}^{T} W^{(i)} R_{i}=I
$$

We can now find the matrix of energy minimizing coarse basis functions $\Psi$ and obtain the coefficient matrix of the coarse space $K_{c}$ as follows

$$
\underbrace{\left[\begin{array}{cc}
K & C^{t} \\
C & 0
\end{array}\right]}_{K_{\mathrm{BIG}}}\left[\begin{array}{c}
\Psi \\
\Lambda
\end{array}\right]=\left[\begin{array}{c}
0 \\
R_{c}
\end{array}\right], \quad K_{c}=\Psi^{T} K \Psi
$$

Finally, the BDDC preconditioner is formulated as

$$
\begin{gathered}
P_{\mathrm{BDDC}}=P_{1}+\left(I-P_{1} A\right) P_{2}\left(I-A P_{1}\right), \\
P_{1}=R_{I}^{T} A_{I I}^{-1} R_{I}, \quad P_{2}=R^{T} W\left(\Psi K_{c}^{-1} \Psi^{T}+P_{3}\right) W R,
\end{gathered}
$$

where $P_{3}$ is defined by

$$
\left[\begin{array}{cc}
K & C^{t} \\
C & 0
\end{array}\right]\left[\begin{array}{c}
P_{3} v \\
\lambda
\end{array}\right]=\left[\begin{array}{l}
v \\
0
\end{array}\right], \quad \forall v
$$

For more details of the formulation and implementation of the BDDC method, we refer the reader to Dohrmann [2003, 2007], Badia et al. [2014].

\section{Perturbed BDDC}

Preconditioner formulation. Let $\widetilde{K}=\operatorname{diag}\left(\widetilde{K}^{(1)}, \ldots, \widetilde{K}^{(N)}\right)$ be a perturbation of $K$. Assume that $\widetilde{K}$ satisfies the following assumptions: 
Assumption 1 There exists two constant $C_{\mathrm{L}}$ and $C_{\mathrm{U}}$ which are independent of the size of the domain (d), the size of the subdomains $(H)$, and the number of the subdomains $(N)$ such that

$$
C_{\mathrm{L}} v^{T} K v \leq v^{T} \widetilde{K} v \leq C_{\mathrm{U}} v^{T} K v, \quad \text { for all } v \text { of appropriate size. }
$$

Assumption 2 The matrix $\widetilde{K}^{(i)}$ is symmetric positive definite (s.p.d) for all $i$.

Assumption 3 There exists a constant $C_{\ell}$ which is independent of the size of the domain (d), the size of the subdomains $(H)$, and the number of the subdomains $(N)$ such that:

$$
C_{\ell} v_{i}^{T} K^{(i)} v_{i} \leq v_{i}^{T} \widetilde{K}^{(i)} v_{i}, \quad \text { for all } v_{i} \text { of appropriate size. }
$$

Let $\widetilde{\Psi}, \widetilde{K}_{c}, \widetilde{P}_{3}$ be defined similarly to $\Psi, K_{c}, P_{3}$ as in (3) and (6), but with $K$ replaced by $\widetilde{K}$. Then the perturbed BDDC preconditioner is given as

$$
\begin{gathered}
\widetilde{P}_{\mathrm{BDDC}}=P_{1}+\left(I-P_{1} A\right) \widetilde{P}_{2}\left(I-A P_{1}\right), \\
\widetilde{P}_{2}=R^{T} W\left(\widetilde{\Psi} \widetilde{K}_{c}^{-1} \widetilde{\Psi}^{T}+\widetilde{P}_{3}\right) W R,
\end{gathered}
$$

Remark 1. If Assumption 2 holds, the matrix $\widetilde{K}$ is s.p.d. From (3), it follows that the coarse matrix $\widetilde{K}_{c}$ is also s.p.d, thus is invertible. In addition, (3) and (6) can be solved using positive definite sparse direct solvers when $K$ is replaced by $\widetilde{K}$. Consequently, corner constraints are not required in the perturbed formulation of BDDC.

Choices of perturbation. We present here two practical choices of perturbed local stiffness matrices $\widetilde{K}^{(i)}$. The first one uses $M^{(i)}$, the mass matrix associated with subdomain $\Omega_{i}$ :

$$
\widetilde{K}^{(i)}=K^{(i)}+\frac{1}{d^{2}} M^{(i)}
$$

The second choice is to use

$$
\widetilde{K}^{(i)}=K^{(i)}+\frac{H_{i}^{n-1}}{d^{n}} M_{\Gamma \Gamma}^{(i)}
$$

where $M_{\Gamma \Gamma}^{(i)}$ is the stiffness matrix associated with subdomain $\Omega_{i}$ assembled only for dofs on the interface. We call this choice Robin perturbation because the local Neumann problem in this case can be posed with Robin boundary condition $\left(H_{j}^{n-1} / D^{n}\right) u+\partial u / \partial n_{i}=0$, where $n_{i}$ is the outward normal vector of $\partial \Omega_{i}$.

It is not difficult to verify that the choices of $\widetilde{K}^{(i)}$ in (7) and (8) satisfy Assumption 1, Assumption 2 and Assumption 3 with $C_{\ell}=C_{\mathrm{L}}=1$ and 
$C_{\mathrm{U}}=1+C_{\Omega}$, where $C_{\Omega}$ depends only on the shape of $\Omega$. Details can be found in Badia and Nguyen [submitted].

\section{Convergence results}

In this section, we present (without proofs) two main convergence results of the perturbed BDDC method. For detailed mathematical analysis, we refer the reader to Badia and Nguyen [submitted].

Theorem 4. There exist a positive constant $C$, independent of $h, H, N, C_{\mathrm{U}}$, $C_{\mathrm{L}}$ and $C_{\ell}$ such that

$$
\kappa\left(\widetilde{P}_{\mathrm{BDDC}} A\right) \leq C \frac{\left(C_{\mathrm{U}}\right)^{2}}{C_{\mathrm{L}} \min \left\{C_{\ell}, C_{\mathrm{L}}\right\}}\left(1+\ln \frac{H}{h}\right)^{2}=\frac{\alpha_{\mathrm{M}}}{\alpha_{\mathrm{m}}},
$$

where $\alpha_{\mathrm{m}}=C_{\mathrm{U}}^{-1}$ and $\alpha_{\mathrm{M}}$ is consistently defined.

The proof of this theorem uses the fact that the spectrum of the preconditioned matrix of the whole system $\widetilde{P}_{\mathrm{BDDC}} A$ is the same as the the spectrum of the preconditioned matrix of the Schur complement $\widetilde{B}_{\mathrm{BDDC}} S$ plus additional eigenvalues equals 1, cf. Dohrmann [2007], Li and Widlund [2006]. The estimates for eigenvalues in the spectrum of $\widetilde{B}_{\mathrm{BDDC}} S$ is documented in detail in Badia and Nguyen [submitted].

Remark 2. Theorem 4 indicates that the perturbed BDDC method has the same polylogarithmic bound for the condition number as the standard one. The precondition number depends on the local problem size but not on the number of subdomains. In other word, the method is weakly scalable.

In order to be well-posed, the standard BDDC method need to have enough constraints to exclude all subdomain-wise constant functions for Poisson's equation and all rigid body modes for linear elasticity. This is no longer necessary for the perturbed BDDC method as its well-posedness is automatically guaranteed. However, the perturbed BDDC method still need to have sufficient constraints to achieve fast convergence.

The following theorem concerns the spectrum of the preconditioned system of the perturbed BDDC method when not all the subdomain-wise constant functions or the rigid body modes are excluded.

Theorem 5. Assume that $\operatorname{ker}\left(K_{\mathrm{BIG}}\right) \neq \emptyset$ then the spectrum of the preconditioned system, counting multiplicities, can be decomposed as

$$
\sigma\left(\widetilde{P}_{\mathrm{BDDC}} A\right)=\mathcal{A}_{1} \cup \mathcal{A}_{2},
$$

where $\left|\mathcal{A}_{1}\right| \leq \operatorname{dim}\left(\operatorname{ker}\left(K_{\mathrm{BIG}}\right)\right), \mathcal{A}_{1} \subset\left[\alpha_{m}, \hat{\alpha}_{M}\right]$ and $\mathcal{A}_{2} \subset\left[\alpha_{m}, \alpha_{M}\right]$. Here, the constants $\alpha_{\mathrm{m}}$ and $\alpha_{\mathrm{M}}$ are defined in Theorem 4, and $\hat{\alpha}_{M}>\alpha_{M}$. 
Remark 3. When the constraints fail to eliminate a small number of subdomainwise constant functions or rigid body modes, namely $\operatorname{ker}\left(K_{\mathrm{BIG}}\right) \neq \emptyset$ and $\operatorname{dim}\left(\operatorname{ker}\left(K_{\mathrm{BIG}}\right)\right)$ is small, Theorem 5 indicates that most of the eigenvalues of the preconditioned system can still be bounded by the usual bounds as in the case with sufficient constraints. Some of the remaining eigenvalues might be larger than the usual upper bound. However, they are isolated (the number of them is bounded from above by $\operatorname{dim}\left(\operatorname{ker}\left(K_{\mathrm{BIG}}\right)\right)$. As large isolated eigenvalues can only delay the convergence of the CG method by few iterations, cf. Axelsson and Lindskog [1986], the perturbed BDDC method is still scalable.

\section{Numerical Experiments}

Both the standard and the perturbed BDDC preconditioners with different options of constraints will be used to solve (2) by the CG method. The number of CG iterations and the time (in second) to reduce the residual by at least a factor of 1e-6 will be reported.

In figures, legends $\mathrm{C}, \mathrm{E}$ and $\mathrm{F}$ are used to indicate corner, edge and face constraints, respectively. The suffix 0 is for the standard BDDC formulation (no perturbation). The suffix CD is to emphasize that the corner selection algorithm by Šístek et al. [2012] and the standard BDDC formulation are used. If the legend is without a suffix, it represents a result with a perturbed BDDC formulation and that no corner selection algorithm is involved.

We present only results for perturbation by full mass matrices. For results using a Robin perturbation, we refer to Badia and Nguyen [submitted]. It is worth noting that the results of the two choices are very close.

We consider (1) with $\Omega$ being the unit cube and elasticity of a beam $\left[\begin{array}{ll}0 & 2\end{array}\right] \times\left[\begin{array}{ll}0 & 0.5\end{array}\right] \times\left[\begin{array}{ll}0 & 0.5\end{array}\right]$. For the latter, (homogeneous) Dirichlet boundary condition is only imposed on one side of the beam (the plane $x=0$ ).

We use uniform structured hexahedral meshes which are partitioned into $k \times k \times k, k=3, \ldots, 11$ (Poisson's problem) and $4 k \times k \times k, k=2, \ldots, 11$ (elasticity) cubic subdomains. For weak scability tests, when $k$ increases ( $H$ decreases), we use smaller mesh size, $h$, to keep $H / h$ constant.

From Fig. 1 and Fig. 2, we can conclude that the perturbed BDDC method, for all the considered choices of constraints, is weakly scalable, namely the numbers of iterations are almost constant when the number of subdomains increases. The performance of the perturbed BDDC method in both iteration number and time are also very close to those of the standard BDDC method.

Among different choices of constraints, the ones with larger coarse spaces, cf. Fig. 3, requires fewer number of iterations, as expected. However, when $N$, the number of subdomains is large, options with smaller coarse spaces, such as $\mathrm{E}$ or F, perform better in time. This is due to the fact that the size of the coarse problem increases as $N$ increases. Consequently, when $N$ increases, the cost of solving the coarse problem become more and more dominant and 
eventually dictates the time performance as coarse tasks and fine tasks are overlapped in advanced implementation of BDDC methods, cf. Badia et al. [2014]. This phenomena exhibits earlier for smaller local problem size $(H / h)$ and options with larger coarse spaces. Therefore, options with edge or/and face constraints only are better suited for solving large scale problems. We emphasize that these options are only available for perturbed BDDC method.

\section{References}

Owe Axelsson and Gunhild Lindskog. On the rate of convergence of the preconditioned conjugate gradient method. Numer. Math., 48(5):499-523, 1986.

Santiago Badia and Hieu Nguyen. Balancing domain decomposition by perturbation. submitted.

Santiago Badia, Alberto F. Martín, and Javier Principe. A highly scalable parallel implementation of balancing domain decomposition by constraints. SIAM J. Sci. Comput., 36(2):C190-C218, 2014.

C. Chevalier and F. Pellegrini. PT-Scotch: A tool for efficient parallel graph ordering. Parallel Computing, 34(68):318 - 331, 2008. Parallel Matrix Algorithms and Applications.

C. R. Dohrmann. An approximate BDDC preconditioner. Numer. Linear Algebra Appl., 14(2):149-168, 2007.

Clark R. Dohrmann. A preconditioner for substructuring based on constrained energy minimization. SIAM J. Sci. Comput., 25(1):246-258, 2003.

George Karypis, Kirk Schloegel, and Vipin Kumar. ParMETIS: Parallel graph partitioning and sparse matrix ordering library. Technical report, Department of Computer Science and Engineering, University of Minnesota, 1997.

Axel Klawonn and Olof B. Widlund. Dual-primal FETI methods for linear elasticity. Comm. Pure Appl. Math., 59(11):1523-1572, 2006.

Michael Lesoinne. A FETI-DP corner selection algorithm for threedimensional problems. In Ismael Herrera, David E. Keyes, Olof B. Widlund, and Robert Yates, editors, Domain Decomposition Methods in Science and Engineering XIV, pages 217-224. National Autonomous University of Mexico (UNAM), Mexico City, Mexico, 2003.

Jing Li and Olof B. Widlund. FETI-DP, BDDC, and block Cholesky methods. Internat. J. Numer. Methods Engrg., 66(2):250-271, 2006.

Jan Mandel. Balancing domain decomposition. Comm. Numer. Methods Engrg., 9(3):233-241, 1993.

Jakub Sístek, Marta Čertíková, Pavel Burda, and Jaroslav Novotný. Facebased selection of corners in 3D substructuring. Math. Comput. Simulation, 82(10):1799-1811, 2012. 

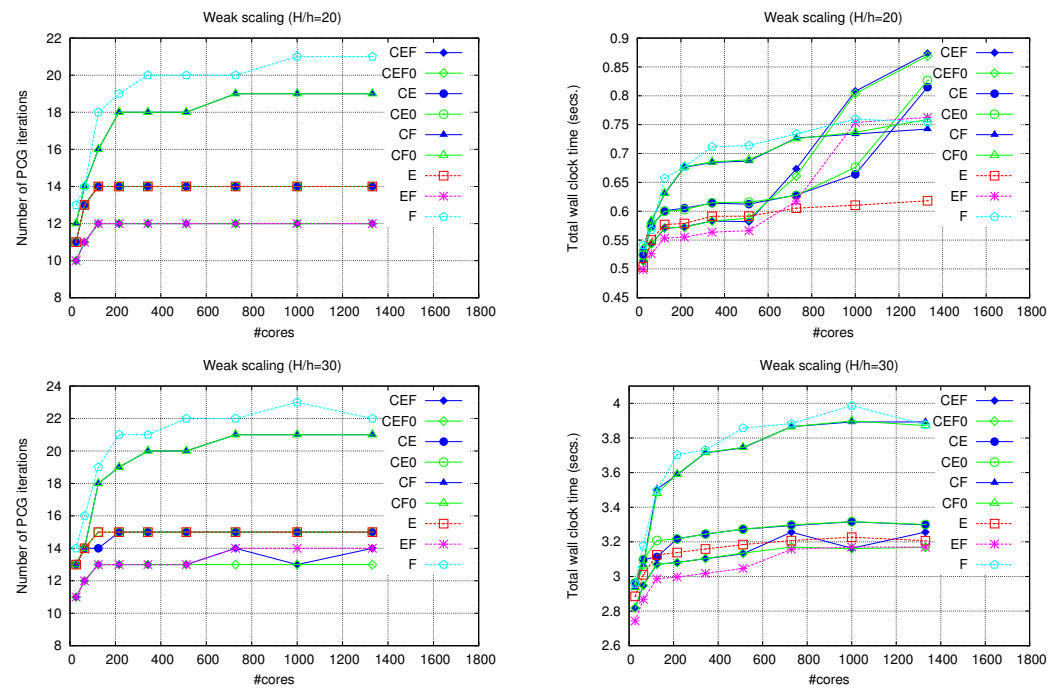

Fig. 1 Poisson's equation: Perturbation with full mass matrices.
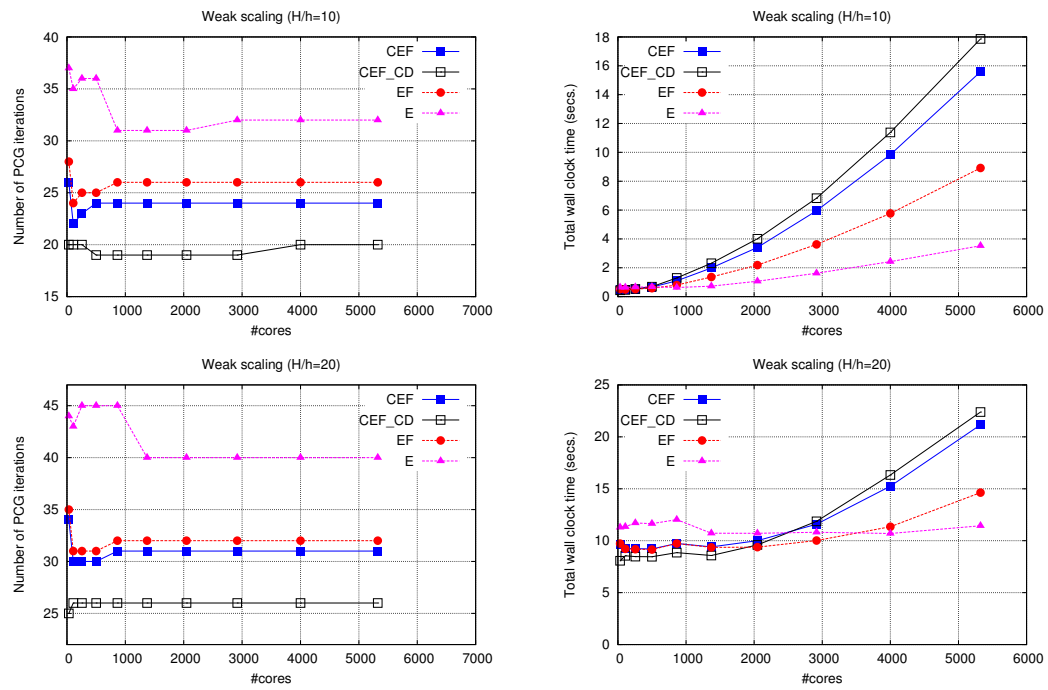

Fig. 2 Elasticity of a beam: Perturbation with full mass matrices.
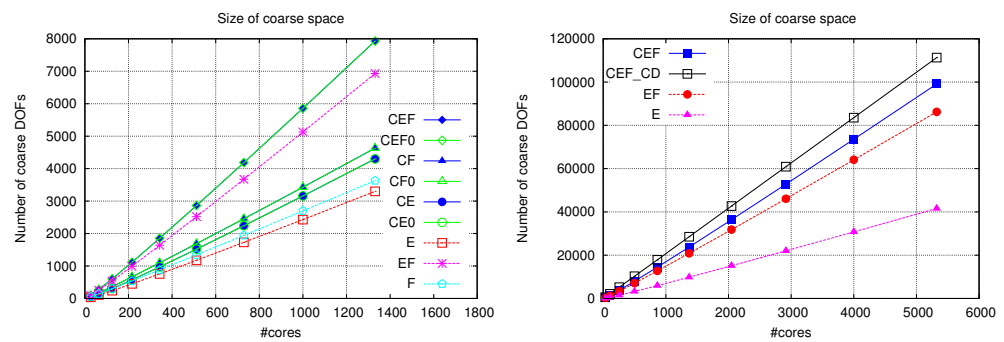

Fig. 3 Size of coarse spaces in Poisson's problem (left) and elasticity problem (right). 